\title{
TDOA and AOA Combined Location Accuracy Analysis with Two Stations
}

\author{
Xinxin Ouyang ${ }^{1,2, *}$, Jingmin Cao ${ }^{1,2}$ and Peiyan Zhao ${ }^{2}$ \\ ${ }^{1}$ University of Electronic Science and Technology of China, Chengdu, Sichuan, China. \\ ${ }^{2}$ National Key Laboratory of Science and Technology on Blind Signal Processing, Chengdu, Sichuan, 86-610041, China \\ ${ }^{*}$ Corresponding author
}

\begin{abstract}
In this paper, a TDOA (Time Difference of Arrival) and DOA (direction of arrival) combined passive location system with two stations is studied. The location of a target can be confirmed from the intersection of the hyperbolic curve defined by the TDOA and the direction ray defined by the DOA. A noniterative approach gives an explicit solution for the proposed system. Based on the model of the location system, the location accuracy is analyzed by calculating the geometric dilution of precision(GDOP), which shows how the factors such as TDOA accuracy and DOA accuracy affect the location accuracy. The results are evaluated by computer simulations.
\end{abstract}

Keywords-component; passive location; time difference of arrival (TDOA);direction of arrival (DOA); geometric dilution of precision(GDOP)

\section{INTRODUCTION}

Passive location has many advantages on anti-stealth, antiradiation missile, anti-extra-low flight, and has been an important aspect of tracking and location research for recent years[1-10]. There are several kinds of passive location system. But hyperbolic location systems and direction finding location systems are the two most important passive location systems, many researches have been done on them. Hyperbolic location systems need three stations with single channel receivers at least, we cannot guarantee that all the stations can receive the target signal in some situation. While direction finding location systems need two stations with multi-channel receivers at least. In some case, there are only a single channel receiver and a multi-channel receiver, thus we have to set up a TDOA and DOA combined location system to complete target localization.

This paper focus on the location accuracy analysis of this system. In section II, we provide a detailed description of the system model, and the explicit solution for the proposed system is given. The influences of TDOA and DOA accuracies are investigated in section III. Section IV gives the computer simulations and the conclusion is in section $\mathrm{V}$.

\section{SYSTEM MODEL}

Figure 1 shows the two-dimensional space model of location system. $\mathrm{S}_{1}$ denotes the station with a multi-channel receiver. We assume that the direction finding antenna is a ULA (uniform linear array) antenna, which gives the DOA of target. While $S_{2}$ denotes the station with a single channel receiver, which gives a hyperbolic curve combined with the $S_{1}$ station when $S_{1}$ and $S_{2}$ is synchronized. T denotes the target, $\theta$ denotes the DOA of target signal, $R$ denotes the distance between $S_{1}$ and $S_{2}$. The intersection of the TDOA hyperbolic curve and the DOA ray is the location of target.

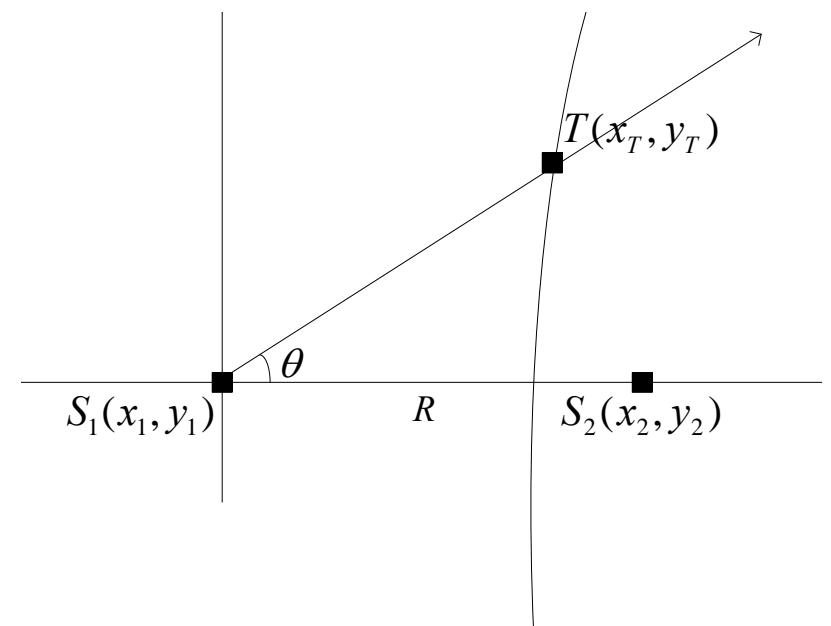

FIGURE I. MODEL OF LOCATION SYSTEM

The coordinate of $\mathrm{S}_{1}$ is $\left(x_{1}, y_{1}\right)$, while the coordinate of $\mathrm{S}_{2}$ is $\left(x_{2}, y_{2}\right)$. The direction of ULA is the same with $R$. The coordinates $\mathrm{T}$ is $\left(x_{T}, y_{T}\right)$. Assuming the TDOA is $\Delta t$ and the velocity of light is $\mathrm{c}$, then

$$
\left\{\begin{array}{l}
R_{T 1}-R_{T 2}=c \Delta t \\
\frac{y_{T}-y_{1}}{x_{T}-x_{1}}=\tan \theta
\end{array}\right.
$$

Where

$$
\left\{\begin{array}{l}
R_{T 1}=\sqrt{\left(x_{T}-x_{1}\right)^{2}+\left(y_{T}-y_{1}\right)^{2}} \\
R_{T 2}=\sqrt{\left(x_{T}-x_{2}\right)^{2}+\left(y_{T}-y_{2}\right)^{2}}
\end{array}\right.
$$

According to the geometric relation, we have 


$$
\cos \theta=\frac{R_{T 1}^{2}+R^{2}-R_{T 2}^{2}}{2 R_{T 1} R}
$$

Put (3) into (1), we can get

$$
R_{T 1}=\frac{R^{2}-c^{2} \Delta t^{2}}{2(R \cos \theta-c \Delta t)}
$$

Introducing $x_{T}=R_{T 1} \cdot \cos \theta, y_{T}=R_{T 1} \cdot \sin \theta$, we obtain that

$$
\left\{\begin{array}{l}
x_{T}=\frac{R^{2}-c^{2} \Delta t^{2}}{2(R \cos \theta-c \Delta t)} \cos \theta+x_{1} \\
y_{T}=\frac{R^{2}-c^{2} \Delta t^{2}}{2(R \cos \theta-c \Delta t)} \sin \theta+y_{1}
\end{array}\right.
$$

\section{ANALYSIS OF GDOP}

When the two observation stations and the target are geometric determined, the location accuracy depends on the estimation accuracy of TDOA and DOA without considering other errors. The partial differential of formula (1) is

$$
\left[\begin{array}{cc}
D_{x} & D_{y} \\
\frac{y_{1}-y_{T}}{\left(x_{T}-x_{1}\right)^{2}} & \frac{1}{\left(x_{T}-x_{1}\right)}
\end{array}\right]\left[\begin{array}{l}
\partial x_{T} \\
\partial y_{T}
\end{array}\right]=\left[\begin{array}{l}
c \partial \Delta t \\
\partial \theta /(\cos \theta)^{2}
\end{array}\right]
$$

where

$$
\left\{\begin{array}{l}
D_{x}=\left(x_{T}-x_{1}\right) / R_{T 1}-\left(x_{T}-x_{2}\right) / R_{T 2} \\
D_{y}=\left(y_{T}-y_{1}\right) / R_{T 1}-\left(y_{T}-y_{2}\right) / R_{T 2}
\end{array}\right.
$$

Assume that

$$
\mathbf{G}_{t}=\left[\begin{array}{cc}
D_{x} & D_{y} \\
\frac{y_{1}-y_{T}}{\left(x_{T}-x_{1}\right)^{2}} & \frac{1}{\left(x_{T}-x_{1}\right)}
\end{array}\right]
$$

and

$$
\boldsymbol{\Phi}=\left(\mathbf{G}_{t}^{T} \mathbf{Q}^{-1} \mathbf{G}_{t}\right)^{-1}
$$

We can obtain that

$$
\begin{aligned}
& \mathbf{Q}=E\left(\left[\begin{array}{c}
c \partial \Delta t \\
\frac{\partial \theta}{(\cos \theta)^{2}}
\end{array}\right]\left[\begin{array}{cc}
c \partial \Delta t & \frac{\partial \theta}{(\cos \theta)^{2}}
\end{array}\right]\right) \\
& =\left[\begin{array}{lc}
c^{2} \sigma_{\Delta t}^{2} & 0 \\
0 & \frac{\sigma_{\theta}^{2}}{(\cos \theta)^{4}}
\end{array}\right]
\end{aligned}
$$

Then the Cramer-Rao lower bound (CRLB) of location error is[2]:

$$
\begin{aligned}
G D O P & =\sqrt{\sigma_{x_{T}}^{2}+\sigma_{y_{T}}^{2}} \\
& =\sqrt{\boldsymbol{\Phi}(1,1)+\boldsymbol{\Phi}(2,2)} \\
& =\sqrt{\frac{\left(x_{T}-x_{1}\right)^{4} \frac{\sigma_{\theta}^{2}}{(\cos \theta)^{4}}\left(D_{x}^{2}+D_{y}^{2}\right)+c^{2} \sigma_{\Delta t}^{2} R_{T 1}^{2}}{\left(D_{x}\left(x_{T}-x_{1}\right)+D_{y}\left(y_{T}-y_{1}\right)\right)^{2}}}
\end{aligned}
$$

\section{SimUlations}

The parameters of simulations are set as follows: the coordinate of $S_{1}$ observation station is $(0,0)$, the single channel receiver stations $S_{2}$ is at $(40 \mathrm{~km}, 0)$. The estimation accuracy of TDOA and DOA depends on the signal parameters such as bandwidth, signal to noise ratio (SNR) and duration time. According to different signals, the estimation accuracy of TDOA is about between 50ns and 500ns, the estimation accuracy of DOA is between $0.1^{\circ}$ and $1^{\circ}$.

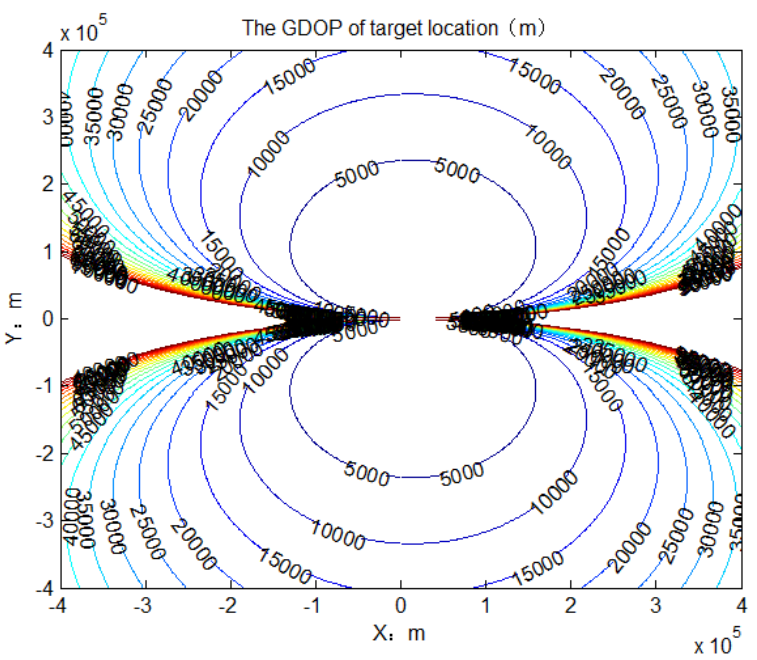

FIGURE II. $\sigma_{T}=50 \mathrm{~ns}, \sigma_{D}=0.1^{\circ}$ 
Figure II to Figure IV show the GDOP under different estimation accuracies of TDOA and DOA. The simulations results show that the location accuracy is affected by the estimation accuracy of DOA and TDOA, but it is affected more by the estimation accuracy of DOA. Figure $\mathrm{V}$ to Figure IV show the length of $\mathrm{R}$ influence the location accuracy.

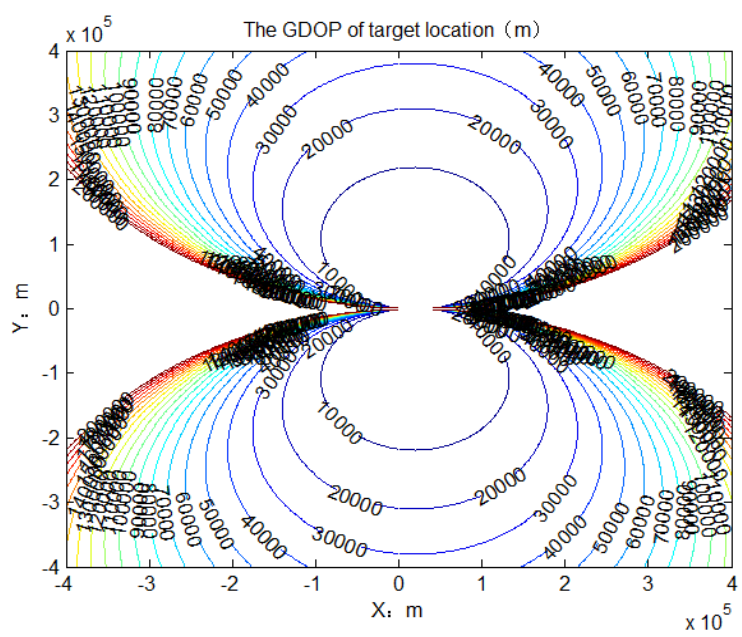

FIGURE III. $\sigma_{T}=500 \mathrm{~ns}, \sigma_{D}=0.1^{\circ}$

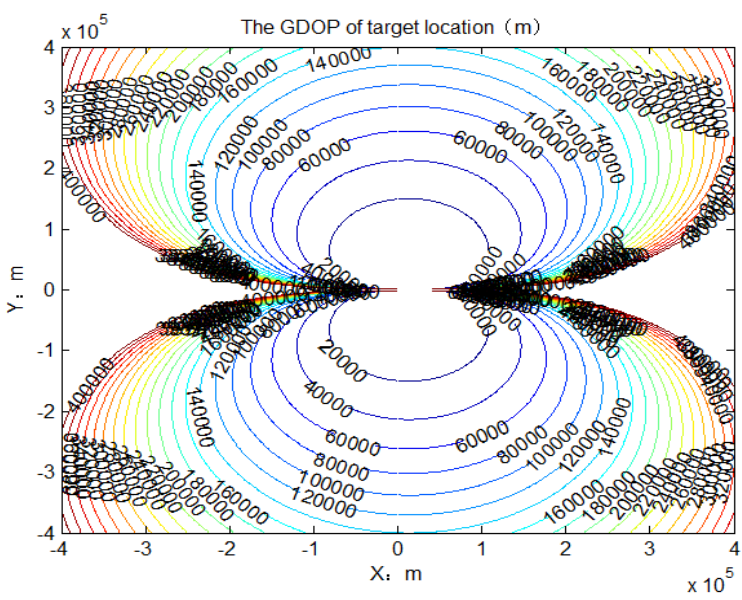

FIGURE IV. $\sigma_{T}=50 \mathrm{~ns}, \sigma_{D}=1^{\circ}$

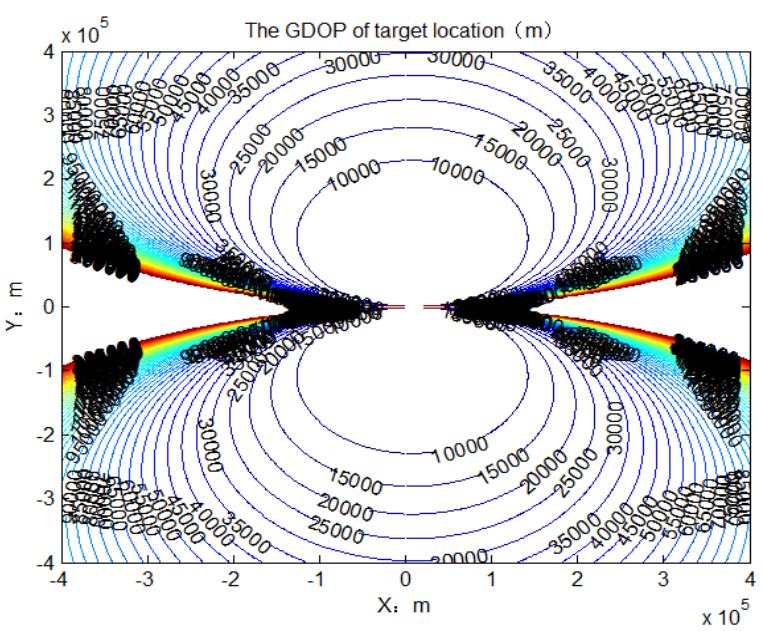

FIGURE V. GDOP for $\sigma_{T}=50 \mathrm{~ns}, \sigma_{D}=0.1^{\circ}, R=20 \mathrm{~km}$

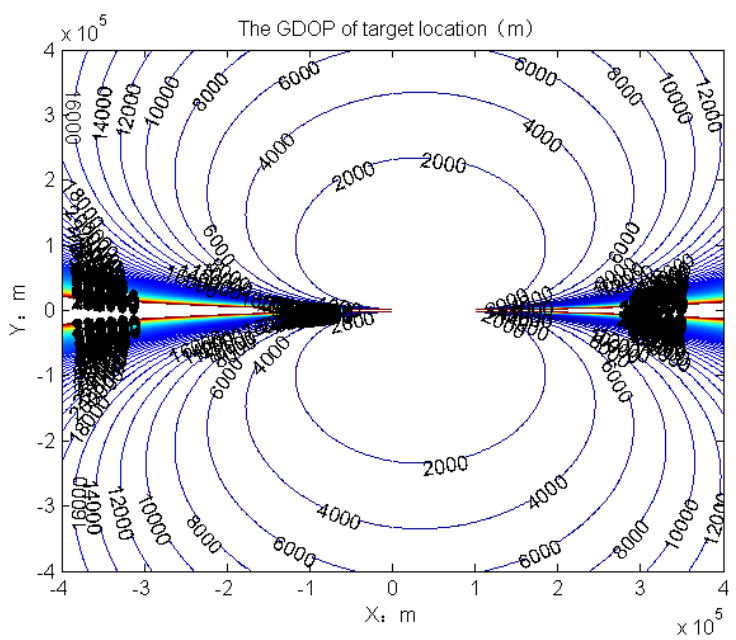

FIGURE VI. GDOP for $\sigma_{T}=50 \mathrm{n} s, \sigma_{D}=0.1^{\circ}, R=100 \mathrm{~km}$

The simulation results of Figure V and Figure VI show that if we want to get higher location accuracy, we should set longer $R$. 


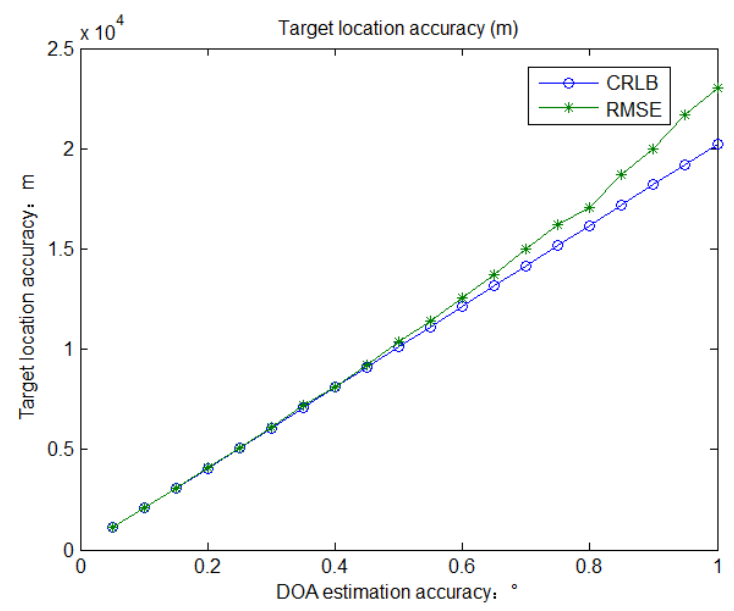

FIGURE VII. LOCATION ACCURACY FOR $\sigma_{T}=50 \mathrm{n} s$

When the target is at $(100 \mathrm{~km}, 100 \mathrm{~km})$, Monte Carlo simulations are provided to show how the DOA and TDOA accuracy affect the location accuracy. Figure VII shows how the DOA estimation accuracy affect the location accuracy when $\sigma_{T}=50 \mathrm{n} s$. While Figure VII shows how the TDOA estimation accuracy affect the location accuracy when $\sigma_{D}=0.1^{\circ}$. From the simulation results, we can also find out that the location accuracy changes following to the DOA and TDOA accuracy, but it changes faster when the DOA accuracy changes. When the TDOA and DOA accuracy is high, the Monte Carlo simulation results are close to the CRLB. It demonstrated the validity of the GDOP derived in the paper.

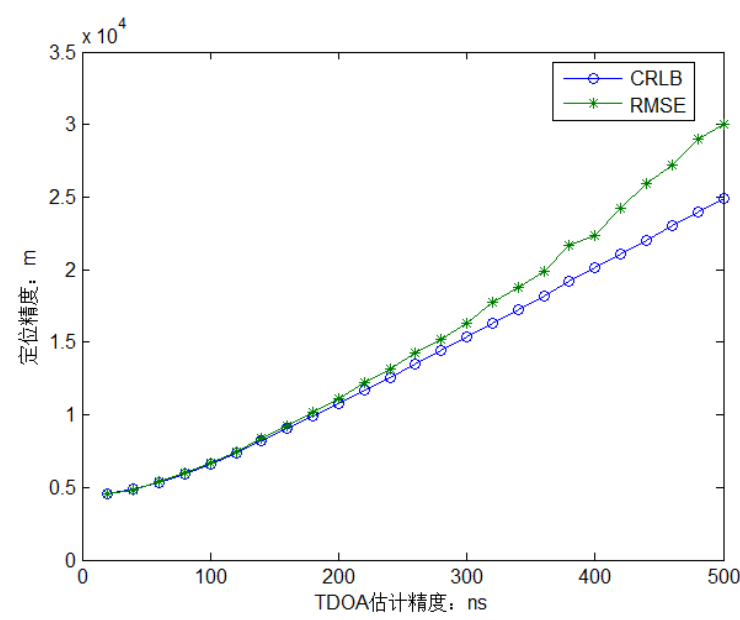

FIGURE VIII. LOCATION ACCURACY FOR $\sigma_{D}=0.1^{\circ}$

\section{CONCLUSION}

In this paper, we investigated a combined passive location system composed by a single channel receiver station and a multi-channel receiver station. Based on the location system model, the influences of TDOA and DOA estimation accuracy are analyzed and the GDOP is given. The theory analysis and simulations show that the system accuracy depends on the accuracy of DOA and TDOA, but depends more on the accuracy of DOA. And the distance between the two stations should set longer to obtain higher location performance.

\section{REFERENCES}

[1] L. Jinzhou, G. Fucheng, Y. Le, J. Wenli, and P. Hongwei. “On the use of calibration sensors in source localization using TDOA and FDOA measurements,” Digital Signal Processing, no.27, pp.33-43, 2014

[2] Y. T. Chan, K. C.Ho, "A simple and efficient estimator for hyperbolic location”, IEEE Transactions on signal processing, 1994, vol.42(8), pp:1905-1915.

[3] H. D. Griffiths and C. J. Baker. Passive coherent location radar systemPart I: performance prediction[J]. IEE Proc - Radar Sonar Navig., 2005,152(3):153-159.

[4] C. J. Baker and H. D. Griffiths. Passive coherent location radar systemPart II: waveform properties[J]. IEE Proc. -Radar Sonar Navig.,2005, 152(3):160-168.

[5] K. W. Cheung, H. C. So, W. K. Ma, et al. A constrained least squares approach to mobile positioning: algorithms and optimality[J]. EURASIP Journal on Applied Signal Processing, 2006, 2006:1-23.

[6] D. J. Torrieri. Statistical theory of passive location system[J]. IEEE Transactions on Aerospace and Electronic Systems, 1984, 20(2):183-197

[7] H. S. Mir, J. D. Sahr, G. F. Hatke, et al. Passive Source Localization Using an Airborne Sensor Array in the Presence of Manifold Perturbations[J]. IEEE Trans. Signal Processing, 2007, 55(6):2486-2496.

[8] B. Q. Huang, L. H. Xie, Z. Yang. TDOA-based source localization with distance-dependent noises[J]. IEEE Transactions on Wireless Communications, 2015, 14(1):468-480.

[9] L. Yang, K. C. Ho. An approximately efficient TDOA localization algorithm in closed-form for locating multiple disjoint sources with erroneous sensor positions[J]. IEEE Trans. Signal Process, 2009, 57(12):4598-4615

Y. H. Kim, D. G. Kim, H. N. Kim. Two-step estimator for movingemitter geolocation using time difference of arrival/frequency-difference of arrival measurements[J]. IET Radar, Sonar \& Navigation, 2015 9(7):881-887 